\title{
Emisiones de compuestos orgánicos volátiles durante arranques en frío de automóviles ligeros
}

\section{Volatile Organic Compound Emissions from Light-Duty Vehicles During Cold-Starts}

\author{
Mendoza-Domínguez A. \\ Departamento de Ingeniería Química \\ Tecnológico de Monterrey, Campus Monterrey, Nuevo León, México \\ E-mail:mendoza.alberto@itesm.mx \\ León-Romero M.A. \\ Centro de Calidad Ambiental \\ Tecnológico de Monterrey, Campus Monterrey, Nuevo León, México \\ E-mail:marco.leon@itesm.mx \\ Caballero-Mata P. \\ Centro de Calidad Ambiental \\ Tecnológico de Monterrey, Campus Monterrey, Nuevo León, México \\ E-mail:pcaballe@itesm.mx
}

(Recibido: septiembre de 2008; reevaluado: julio de 2009; aceptado: febrero de 2010)

\section{Resumen}

El arranque en frío ha sido catalogado como uno de los periodos del ciclo de operación de los vehículos, en donde se puede esperar una cantidad importante de emisiones de compuestos orgánicos volátiles (COVs). En el presente trabajo se reportan los resultados de dos campañas de monitoreo realizadas en el Área Metropolitana de Monterrey, México, para caracterizar las emisiones en el escape de 20 automóviles de modelo reciente (preferentemente de tipo sedán) durante eventos de arranque en frío. Las muestras obtenidas fueron sujetas a análisis químico para identificar el perfil de COVs en las emisiones generadas. El análisis químico estuvo centrado en 30 alcanos y aromáticos en el intervalo C5 a C9. Los resultados revelaron que las especies con mayor concentración fueron: Dimetilhexano, 2,2,4-Trimetilpentano y 2,3,4-Trimetilpentano. Asimismo, se encontró que vehículos que emplean el mismo tipo de gasolina (Magna o Premium) tienden a tener un perfil químico de emisiones parecido y estadísticamente diferente al otro grupo. El efecto del kilometraje recorrido, el año-modelo o la marca del vehículo no fue determinante para este resultado. Esto implica que el perfil químico de las emisiones obtenidas, es prácticamente una función exclusiva del tipo de gasolina empleado y no de las condiciones del vehículo. Por otro lado, las relaciones promedio calculadas entre las emisiones de Benceno, Tolueno y Etilbenceno (T:B-1.9, E:B-0.6 y B:T-1.5) están dentro de los intervalos de referencia encontrados en la literatura. Finalmente, se clasificaron los compuestos emitidos respecto a su reactividad (potencial de formación de ozono) empleando una escala de concentración propileno equivalente. Se 
DOI: http://dx.doi.org/10.22201/fi.25940732e.2010.11n3.029

Emisiones de compuestos orgánicos volátiles durante arranques en frío de automóviles ligeros

encontró que la reactividad de los gases de escape está gobernada por el Dimetilhexanoy el 2,3,4-Trimetilpentano, especies que son la primera y tercera más abundantes. El resto de las especies no necesariamente tienen el mismo lugar en la escala de concentración que en la escala de reactividad.

Descriptores: emisiones vehiculares, contaminación atmosférica, inventarios de emisiones, fuentes móviles, monitoreo de emisiones.

\begin{abstract}
Cold-starts have been catalogued as one of the periods during the operation cycle of vehicles where significant amounts of volatile organic compound (VOC) emissions can occur. In this work we present the results of two monitoring campaigns held in the Metropolitan Area of Monterrey, Mexico, where the exhaust gases of 20 recent-model ligh-duty vehicles were characterized during cold-start events. The collected samples were chemically analysed to obtain the corresponding VOC emission profiles. The chemical analysis focused on 30 alkanes and aromatic species in the range $C 5$ to $C 9$. The results revealed that the species with highest concentrations were Dimethylhexane, 2,2,4-Trimethylpentane, and 2,3,4-Trimethylpentane. Moreover, it was found that vehicles that used the same type of gasoline (Magna or Premium) tended to have a similar chemical profile of their emissions, and statistically different from the other group. The effect of milage, model-year or brand of the vehicle did not affect this result. This implies that the chemical profile of the emissions during cold-starts is practically an exclusive function of the type of gasoline used, and not of the vehicle's conditions. Average ratios between Benzene, Toluene, and Ethylbenzene emissions were also calculated (T:B - 1.9, E:B $0.6 y B: T-1.5)$, and were in the reference ranges found in the literature. Finally, the emitted species were classified according to their reactivity (ozone forming potential) using a propylene-equivalent concentration scale. It was found that the reactivity of the exhaust gases is governed by the first and third most abundant identified species (Dimethylhexane and 2,3,4-Trimethylpentane). The rest of the species do not necessarily have the same position in the concentration scale as in the reactivity scale.
\end{abstract}

Keywords: Vehicle emissions, air pollution, emissions inventory, mobile sources, emissions monitoring.

\section{Introducción}

La contaminación atmosférica se presenta en la mayoría de las grandes ciudades, por lo que se ha convertido en un gran problema que requiere una atención prioritaria, principalmente por los riesgos a la salud que conlleva la exposición a contaminantes atmosféricos tóxicos. Entre los contaminantes atmosféricos que requieren atención se encuentran los compuestos orgánicos volátiles (COVs). Un COV es cualquier compuesto de carbono (exceptuando $\mathrm{CO}, \mathrm{CO}_{2}$, ácido carbónico, carbonatos metálicos y carbonato de amonio) con la suficiente capacidad de participar en reacciones fotoquímicas atmosféricas (Seinfeld, 1998), es decir, con suficiente reactividad fotoquímica para participar en la formación de ozono o de aerosoles secundarios.
Típicamente, los COV son compuestos que tienen una alta presión de vapor $\left(0.01 \mathrm{kPa}\right.$ a $\left.20^{\circ} \mathrm{C}\right)$ y una baja solubilidad en agua. Algunos, incluso, se les ha catalogado como cancerígenos.

Entre los diversos usos que se le dan a los COVs está su presencia en combustibles para motores de combustión interna, como químicos intermedios, como solventes para grasas, tintas, aceites, pinturas, plásticos, etc. Muchos estudios han sido llevados a cabo en diversas ciudades alrededor del mundo con el fin de determinar la fuente principal de dichos compuestos (p. ej., Brocco et al., 1997; Cheng et al., 1997; Grosjean et al., 1998; Varshney et al., 1998; Thijsse et al., 1999; Derwent et al., 2000; Vukovich, 2000; Na y Kim, 2001), incluyendo poblaciones en México, como la Ciudad de México (Mugica et al., 2003) y Mexicali (Mendoza et al., 2008). 
DOI: http://dx.doi.org/10.22201/fi.25940732e.2010.11n3.029

Mendoza-Domínguez A., León-Romero M.A. y Caballero-Mata P.

Los resultados, en general, han mostrado que más del $40 \%$ de los COvs encontrados en el aire ambiente de centros urbanos tiene su fuente principal en las emisiones del escape de fuentes móviles (principalmente vehículos a gasolina), y existe una relación directa entre los niveles de COVs en el aire ambiente y los patrones de tráfico locales (Watson et al., 2001). Así mismo, otros estudios (p. ej., Mugica et al., 2003) han mostrado que la concentración de aromáticos volátiles en cruceros y estacionamientos, es muchas veces mayor que la concentración en el aire ambiente, por lo que existe un riesgo para la salud de los peatones, vendedores ambulantes y policías, siendo la principal fuente de emisión de dichos compuestos aromáticos los vehículos. Los resultados anteriores no son sorprendentes si se considera que las emisiones debidas a fuentes móviles representan un gran porcentaje del total del inventario de emisiones reportados en áreas urbanas. Por ejemplo, en Estados Unidos se estimó que en 1999, las fuentes móviles contribuyeron con aproximadamente $64 \%$ del CO total, $35 \%$ de los $\mathrm{NOx}\left(\mathrm{NO}+\mathrm{NO}_{2}\right)$ totales y $27 \%$ de los COVs emitidos (US EPA, 2001). En México, el inventario de emisiones con año base también de 1999 reportado para los seis estados fronterizos reportó que las fuentes móviles contribuyeron con $72 \%$ del CO emitido, $24 \%$ del NOx y 23\% de los COVs (ERG et al., 2004). Estas cifras incluyen las emisiones de fuentes antropogénicas y biogénicas.

Debido a la importante contribución de las fuentes móviles al total de emisiones de COVs en las ciudades modernas, una apreciable cantidad de estudios se han realizado para entender los perfiles químicos de emisión de dichos compuestos (p. ej., Mugica et al., 1998; Gamas et al., 1999, Corvalan et al., 2000; Díaz et al., 2001, Zielinska et al., 2004). Esto es relevante para conocer no sólo la cantidad total emitida, sino también para conocer el potencial que tienen dichas emisiones en la formación de oxidantes fotoquímicos (particularmente ozono), aerosoles secundarios, y la cantidad de tóxicos (potencialmente cancerígenos) emitidos.

Los vehículos tienen diferentes tasas de emisión dependiendo del ciclo de manejo en el que se encuentren: arranque (ya sea en frío o en caliente), tránsito (aceleración, frenado, paro con el motor en marcha, velocidad estable). Asimismo, también se producen emisiones evaporativas cuando el vehículo está detenido, con o sin el motor en marcha, o en movimiento. De todas estas condiciones, se ha estimado que durante los arranques en frío se pueden llegar a producir emisiones de COVs mucho mayores que cuando el vehículo está en marcha. Por ejemplo, Graham et al. (2004) demostraron que las emisiones en el escape de vehículos durante períodos de arranque pueden ser mayores para el arranque en frío que para el arranque en caliente, en particular: 32 veces más para el $\mathrm{CO}$, el doble para el $\mathrm{CO}_{2}, 10$ veces más para NOx y 18 veces más para los hidrocarburos no quemados. Asimismo, la composición de los gases emitidos, particularmente de los COVs, es diferente en los dos diferentes tipos de arranque (Singer et al., 1999). Por ello, es importante caracterizar químicamente las emisiones durante los periodos de arranque en frío del parque vehicular de ciudades mexicanas, y con ello, tener un mejor estimado de las emisiones a través de factores de emisión más precisos. Esta información es de gran valor, ya que eventualmente los inventarios de emisiones y los perfiles de emisión se emplean en estudios de modelación atmosférica (inclyendo modelos receptor) e impactos a la salud (exposición y riesgo). Así, el objetivo del presente trabajo es la obtención de una caracterización química de los COVs presentes en las emisiones durante los periodos de arranques en frío de vehículos de modelo reciente del parque vehicular del Área Metropolitana de Monterrey (AMM), México.

\section{Emisiones por fuentes móviles durante arranques en frío}

Las emisiones de COVs, COy NOx provenientes del escape de vehículos automotor han sido abatidas sustancialmente desde finales de los 60's, debido a la implementación de controles tecnológicos que reducen la formación de contaminantes durante la combustión y remueven contaminantes de gases de salida del escape (Singer et al., 1999). Un estricto control estequiométrico de la relación aire-combustible resulta en menores niveles de producción de $\mathrm{CO}$ y COVs relacionados a la operación con exceso de combustible. El control de la relación aire-combustible también resulta en bajos niveles de producción de NOx relacionados con la operación con deficiencia de combustible. Las mezclas estequiométricas aire-combustible también son requeridas en el tratamiento de gases de salida por parte de los convertidores catalíticos de tres vías, los cuales oxidan simultáneamente los $\mathrm{COV}$ s y el $\mathrm{CO}$ a $\mathrm{CO}_{2}$ y reducen los $\mathrm{NOx}$ a $\mathrm{N}_{2}$. Una limitación de los sistemas de control actuales es que son ineficientes por un corto periodo de tiempo después del arranque del vehículo. En el arranque, la mezcla aire-combustible está intencionalmente excedida de combustible con el fin de facilitar la ignición y mejorar la operación del motor en frío. Este exceso lleva a un incremento en la producción de COVs y CO durante la combustión y limita la oxidación de estos 
DOI: http://dx.doi.org/10.22201/fi.25940732e.2010.11n3.029

Emisiones de compuestos orgánicos volátiles durante arranques en frío de automóviles ligeros

contaminantes en el convertidor catalítico. Además, los convertidores catalíticos de los automóviles necesitan alcanzar temperaturas entre los $200^{\circ} \mathrm{C}-400^{\circ} \mathrm{C}$ para lograr una conversión significativa de los contaminantes (Heywood, 1988). Es importante mencionar que el convertidor catalítico depende del calor de los gases de salida para calentarse hasta alcanzar la temperatura de operación. Conforme el convertidor se va calentando, su eficiencia de conversión se va incrementando hasta que alcanza la eficiencia máxima a temperaturas normales de operación. Es por ello que resulta de gran importancia e interés la caracterización de los gases de salida del tubo de escape de los automóviles en estas condiciones de operación.

\section{Metodología de muestreo}

Técnica de muestreo

Para el muestreo de gases en el tubo de escape y su posterior análisis químico se utilizó una bomba de succión (Gast Corporation MFG, modelo DAA-VI2G6) conectada directamente a una sonda de muestreo. En cada prueba y previo al arranque del vehículo, la bomba se encendió para ajustar el flujo de salida de la bomba y cumplir con el tiempo de muestreo preseleccionado. Después de dicho ajuste, se colocó la sonda a la salida del tubo de escape del vehículo a evaluar, se encendió el vehículo para iniciar el muestreo y se procedió a esperar 15 segundos para que se purgara la línea del tubo de escape. Posteriormente se colocó una bolsa Tedlar de $12 \times$ 12 in y capacidad de $5 \mathrm{~L}$, justo en el tubo de salida de la bomba para iniciar el llenado de las mismas. Una vez colectada la muestra, la bolsa Tedlar se guardó dentro de una bolsa de plástico negra para proteger la muestra de los rayos solares y evitar que se produjeran reacciones químicas indeseables que alteraran el contenido de la bolsa. El análisis químico se efectuó en todos los casos el mismo día que se tomaba la muestra para evitar degradación del contenido.

Respecto al tiempo de duración de toma de muestra, en la literatura se hace referencia de un tiempo estimado de 250-300 segundos para el ciclo de arranque en frío (Singer et al., 1999; Graham et al., 2004) en el cual los perfiles de emisiones comienzan con picos agudos de hidrocarburos (HC) y CO, resultado del exceso de combustible para la ignición. Sin embargo, es en un periodo de entre 90-110 segundos posterior al momento mismo del arranque donde se registra la máxima emisión acumulada para este tipo de arranque (Graham et al., 2004). Las emisiones de $\mathrm{CO}$ y $\mathrm{HC}$ alcanzan rápidamente su pico y posteriormente caen conforme se va calentando el convertidor. Después de este periodo las emisiones de HC y CO permanecen bajas y constantes durante la extensión del periodo inactivo, que empieza a los 125 segundos. Para los 200-220 segundos, el convertidor ya alcanzó altas temperaturas estables y las emisiones de HC y CO llegan a sus valores más bajos (US EPA, 1993). Tomando en cuenta lo anterior, se definió un ciclo de muestreo que va desde los 15 segundos hasta los 105 segundos, con el fin de captar la concentración máxima acumulada de contaminantes. Finalmente, en todos los casos el vehículo probado debió permanecer sin actividad mínimo por 10 horas antes de realizar el muestreo; los muestreos preferentemente ocurrieron en el periodo de la mañana.

\section{Análisis químico de las muestras}

Para el análisis químico de las muestras tomadas en las bolsas Tedlar se utilizó un cromatógrafo de gases con espectrometría de masas (GC-MS) HP 5890 Serie II con un sistema de purga y trampa para la inyección de muestras. El método analítico empleado fue el método 8260B de la Agencia de Protección Ambiental de los EE.UU. (US EPA, por sus siglas en inglés). Este método es aplicable prácticamente a todos los tipos de muestras, sin importar el contenido de agua. Esto incluye varios medios de trampeo de muestras de aire, suelo y agua superficial, lodos acuosos, licores cáusticos, licores ácidos, solventes residuales y residuos aceitosos. En el caso de este estudio se inyectaron $15 \mathrm{~mL}$ de muestra directamente a la columna capilar y se cuantificaron las respuestas respecto a un estándar interno usando una curva de calibración de cinco puntos.

Respecto a la lista de compuestos identificados en el análisis químico, ésta se generó después de una revisión de los perfiles de emisión que se obtuvieron en varios estudios realizados tanto en México como en otros países (Mugica et al., 2001; Gamas et al., 1999; Ho et al., 1998; Singer et al., 1999; Graham et al., 2004). Además de estas caracterizaciones, también se tomaron en cuenta los perfiles obtenidos en estudios de aire ambiente en la Ciudad de México (Mugica et al., 2003) y en otras regiones del orbe (Mukerjee et al., 2004; Thijssee et al., 1999). Así también, se consideraron las limitantes impuestas por el equipo disponible de análsis químico (principalmente tipo de columna capilar disponible en el equipo de cromatografía) y otras limitantes en disponibilidad de recursos. Tomando en cuenta lo anterior, la lista de especies propuestas para el análisis es la que se presenta en la tabla 1, y está compuesta principalmente de alcanos en el intervalo C5 a C9 y de aromáticos en el intervalo C6 a C9. 
DOI: http://dx.doi.org/10.22201/fi.25940732e.2010.11n3.029

Mendoza-Domínguez A., León-Romero M.A. y Caballero-Mata P.

Tabla 1. Especies analizadas en los gases de escape recolectados durante las pruebas de arranque en frío

\begin{tabular}{ccc}
\hline & Alcanos & Aromáticos \\
\hline Isobutano & n-Hexano & Benceno \\
2,2-DMB (a) & Ciclohexano & Tolueno \\
2,3-DMB & Dimetilhexano $\left(^{*}\right)$ & Etilbenceno \\
Isopentano & 2-Metil-Hexano & o-Xileno \\
$n$-Pentano & 3-Metil-Hexano & $m$-Xileno \\
Ciclopentano & $n$-Heptano & -Xileno \\
2-metilpentano & $n$-Nonano & Cumeno \\
3-metilpentano & & $1,3,5$-TMB (d) \\
2,3-DMP (b) & & $1,2,4$-TMB (d) \\
2,2,4-TMP (c) & & $n$-Propilbenceno \\
2,3,4-TMP (c, ${ }^{(\text {) }}$ & & Estireno \\
\hline
\end{tabular}

a DMB: Dimetilbutano; b DMP: Dimetilpentano; ${ }^{\mathrm{c}}$ TMP: Trimetilpentano;

d TMB: Trimetilbenceno.

* Estos compuestos no fueron calibrados para su análisis, se semicuantificaron por

medio de una curva de calibración de un compuesto químicamente similar

Lote vehicular estudiado

El estudio se realizó en dos campañas de monitoreo: la primera de ellas se llevó a cabo del 28 de abril al 2 de mayo de 2005, mientras que la segunda se realizó del 27 al 30 de septiembre de 2005. En ambas ocasiones los muestreos se realizaron en automóviles ligeros, en su mayoría tipo sedán, de diversas marcas y modelos (preferentemente modelos recientes: 2003-2005), que contaban a lo más con seis cilindros y estuvieran registrados en el Estado de Nuevo León (preferentemente que circulaban cotidianamente en el AMM). Un total de 20 automóviles fueron empleados (tablas 2 y 3 ), cinco de ellos siendo diferentes entre ambas campañas, debido a que durante el lapso de tiempo entre los muestreos, dichos vehículos cambiaron de dueño o localización geográfica haciendo imposible su muestreo.

Del total de vehículos empleados, $60 \%$ de ellos reportaron un kilometraje menor a los $30,000 \mathrm{~km}$ al momento de efectuar las pruebas y solamente un vehículo excedió los $60,000 \mathrm{~km}$. Asimismo, el 60\% de los vehículos monitoreados fueron de modelos recientes (años 2004 y 2005), mientras que un $75 \%$ de los automóviles se ubican dentro del periodo 2002-2005.

Por otro lado, las marcas encontradas con mayor frecuencia en el lote de automóviles empleado fueron Nissan (30\%) y Chevrolet (25\%). Los vehículos se seleccionaron aleatoriamente y, sobre todo, con base a la disponibilidad de los mismos para el desarrollo de este proyecto. Comparando los porcentajes de distribución de marcas usadas en este estudio con respecto a los reportados para el parque vehicular del Estado de Nuevo León en un estudio previo (Quesada, 2003), se encontró una distribución parecida para las marcas Chevrolet, VW y Chrysler (25\%, 15\% y $10 \%$ contra $22 \%, 14 \%$ y $11 \%$, respectivamente), mientras que para las marcas Nissan y Ford fue notable la diferencia ( $30 \%$ vs $12 \%$ para Nissan y $5 \%$ vs $24 \%$ para Ford). Cabe señalar que en el estudio llevado a cabo por Quesada (2003) se obtuvieron los porcentajes antes citados, tomando en cuenta vehículos de todos los modelos en el registro del Estado de Nuevo León del año 2003. Pese a que el lote de vehículos empleado en este trabajo fue seleccionado con base a disponibilidad, resultó tener una representatividad aceptable de marcas, respecto a la distribución de vehículos que típicamente se encuentran circulando en el Estado. Por otro lado, el parque vehicular del AMM representa aproximadamente un $87 \%$ del total del parque vehicular del Estado (Quesada, 2003), por lo que es de esperarse que la distribución de marcas sea parecida entre uno y otro parque, y por ende, también se tendría cierto grado de representatividad en distribución de marcas entre el lote de vehículos usados en este estudio con el del parque vehicular del AMM. 
DOI: http://dx.doi.org/10.22201/fi.25940732e.2010.11n3.029

Emisiones de compuestos orgánicos volátiles durante arranques en frío de automóviles ligeros

Tabla 2. Datos generales de los automóviles monitoreados durante la primera campaña

\begin{tabular}{|c|c|c|c|c|}
\hline Identificador & Kilometraje & Marca / Tipo & Gasolina & Modelo \\
\hline$M-1$ & 19,872 & Nissan / X-Trail & Magna & 2004 \\
\hline$M-2$ & 8,071 & Chevrolet / Corsa & Premium & 2004 \\
\hline$M-3$ & 1,885 & Peugeot / Peugeot 206 & Premium & 2005 \\
\hline$M-4$ & 53,012 & Chevrolet / Cavalier & Magna & 2000 \\
\hline$M-5$ & 5,661 & Chevrolet / Astra & Magna & 2005 \\
\hline$M-6$ & 15,700 & Peugeot / Peugeot 206 & Premium & 2002 \\
\hline$M-7$ & 13,322 & Nissan / Platina & Premium & 2004 \\
\hline M-8 & 33,043 & Nissan / Tsuru & Magna & 2003 \\
\hline M-9 & 52,279 & VW / Jetta & Magna & 2004 \\
\hline$M-10$ & 16,000 & VW / Pointer City & Premium & 2004 \\
\hline$M-11$ & 22,258 & Renault / Megane & Premium & 2004 \\
\hline$M-12$ & 48,743 & Chevrolet / Sunfire & Magna & 1999 \\
\hline$M-13$ & 15,814 & Nissan / Tsuru & Premium & 2004 \\
\hline$M-14$ & 59,738 & Chevrolet / Chevy & Magna & 2000 \\
\hline$M-15$ & 5,180 & Nissan / Platina & Premium & 2005 \\
\hline
\end{tabular}

Tabla 3. Datos generales de los automóviles monitoreados durante la segunda campaña

\begin{tabular}{|c|c|c|c|c|}
\hline $\begin{array}{c}\text { Identificador } \\
\text { (a) }\end{array}$ & Kilometraje & Marca & Gasolina & Modelo \\
\hline AUTO -1 & 84,761 & VW / Jetta & Magna & 2001 \\
\hline AUTO -2 & 27,211 & Chrysler / Neon & Magna & 2004 \\
\hline AUTO -3 & 48,243 & Chrysler / Neon & Magna & 2002 \\
\hline CAM-1 & 300 & Ford / Ecosport & Premium & 2005 \\
\hline CAM-2 & 40,736 & Nissan / Estaquitas & Magna & 2001 \\
\hline$M-2$ & 11,132 & Chevrolet / Corsa & Premium & 2004 \\
\hline M-3 & 7,845 & Peugeot / Peugeot 206 & Premium & 2005 \\
\hline$M-4$ & 64,852 & Chevrolet / Cavalier & Magna & 2000 \\
\hline M-5 & 11,117 & Chevrolet / Astra & Magna & 2005 \\
\hline$M-10$ & 17,060 & VW / Pointer City & Premium & 2004 \\
\hline$M-11$ & 35,880 & Renault / Megane & Premium & 2004 \\
\hline$M-12$ & 60,413 & Chevrolet / Sunfire & Magna & 1999 \\
\hline$M-13$ & 21,526 & Nissan / Tsuru & Premium & 2004 \\
\hline$M-14$ & 67,112 & Chevrolet / Chevy & Magna & 2000 \\
\hline$M-15$ & 5,280 & Nissan / Platina & Premium & 2005 \\
\hline
\end{tabular}

a Los vehículos con identificadores M-X corresponden a los mismos listados en la tabla 2. 
DOI: http://dx.doi.org/10.22201/fi.25940732e.2010.11n3.029

Mendoza-Domínguez A., León-Romero M.A. y Caballero-Mata P.

\section{Resultados}

Perfil químico de emisiones

Los análisis químicos que se realizaron a las muestras colectadas durante las dos campañas de monitoreo arrojaron datos de concentración para 14 COVs (figura 1). En todos los casos, el límite de detección estimado del método empleado fue menor a $10 \mathrm{ng} / \mathrm{mL}$. Los valores presentados representan el promedio de todas las muestras tomadas, no importando el tipo de gasolina empleado. Las especies con mayor concentración promedio fueron: Dimetilhexano (181.5 ng/mL), 2,2,4-Trimetilpentano $(176.0 \mathrm{ng} / \mathrm{mL}$ ) y 2,3,4-Trimetilpentano (154.4 $\mathrm{ng} / \mathrm{mL}$ ). Gamas et al. (1999) también reportan en su estudio sobre emisiones de fuentes móviles realizado en la Ciudad de México una alta contribución de 2,2,4- y 2,3,4-Trimetilpentano al total de COVs obervados.

\section{Correlaciones entre especies químicas}

Con los datos obtenidos de la caracterización química, se realizó un análisis de correlación entre todas las especies identificadas, empleando el coeficiente de correlación de Pearson, $r$ (correlación lineal). Como referencia, se tomó que una buena correlación se obtenia cuando el valor de $r^{2}$ (coeficiente de deteterminación) oscilaba entre 0.80 y 1.0 , era moderada cuando el valor de $r^{2}$ estaba entre 0.5 y 0.8 , mientras que una correlación se consideró débil para valores de $r^{2}$ inferiores a 0.50 (Devore, 2000). La tabla 4 muestra un resumen de las correlaciones promedio obtenidas y catalogadas como "buenas" o "moderadas". Cabe mencionar que a manera de verificación de la información de la asociación entre especies químicas analizadas inferida por el coeficiente de correlación de Pearson, también se calculó el coeficiente de correlación de Spearman (prueba no paramétrica) obteniéndose resultados consistentes entre ambas pruebas. El coeficiente de correlación de Spearman no se reporta por brevedad.

Entre compuestos alifáticos, el 2-Metil-Pentano, el Ciclohexano, el Dimetilhexano, el 2,3,4-TMP y el 2,2,4-TMP son los que reportaron una mayor correlación entre ellos, siendo ésta alta en nueve de los 10 casos presentados en la tabla 4 (moderada entre 2-Metil-Pentano y Ciclohexano). Entre compuestos aromáticos, el $m, p$-Xileno fue la especie que se relacionó mejor con las demás (alta en 3 de los 4 casos), seguida del Benceno y el Etilbenceno (una correlación alta y una moderada). Finalmente, en las correlaciones cruzadas entre alifáticos y aromáticos, el o-Xileno fue la especie que mejor se relacionó con los alifáticos ( 3 correlaciones altas y una moderada); el Etilbenceno tuvo dos casos de correlación moderada. Las variaciones en la correlación entre especies puede deberse al combustible empleado por el

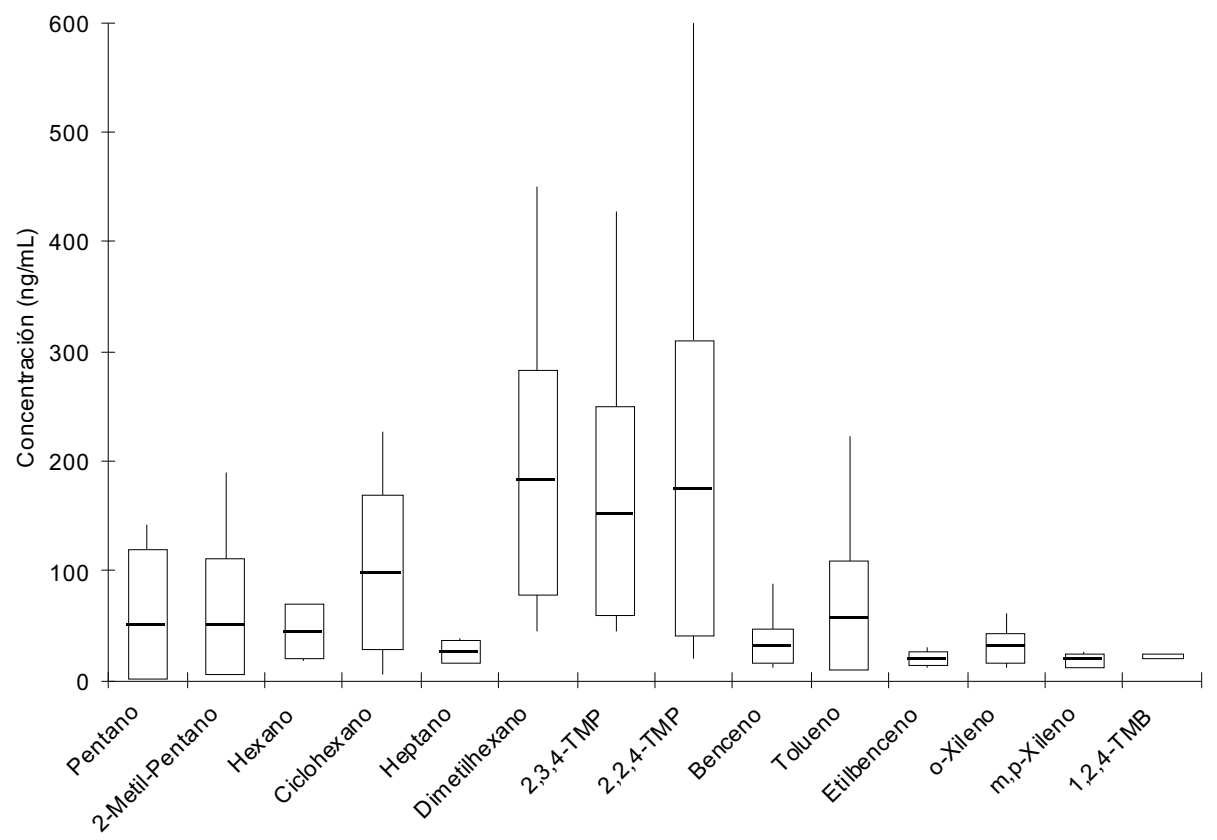

Figura 1. Concentración promedio de COVs en los gases de escape. La gráfica ilustra el promedio \pm una desviación estándar dentro del recuadro de cada compuesto, y el máximo y minimo indicados por los extremos de las líneas correspondientes 
DOI: http://dx.doi.org/10.22201/fi.25940732e.2010.11n3.029

Emisiones de compuestos orgánicos volátiles durante arranques en frío de automóviles ligeros

Tabla 4. Valores $r^{2}$ para las parejas de especies en las cuales se obtuvo una correlación lineal alta o moderada

\begin{tabular}{cccc}
\hline Correlaciones altas $\left(r^{2}>0.8\right)$ & & Correlaciones moderadas $\left(0.5<r^{2}<0.8\right)$ \\
Especies químicas & $r^{2}$ & Especies químicas & $r^{2}$ \\
\hline o-Xileno / m,p-Xileno & 1.000 & 2,3,4-TMP / Etilbenceno & 0.794 \\
Ciclohexano / 2,2,4-TMP & 0.998 & Etrilbenceno / o-Xileno & 0.783 \\
Etilbenceno / m,p-Xileno & 0.995 & Dimetilhexano / Etilbenceno & 0.770 \\
Ciclohexano / Dimetilhexano & 0.983 & Heptano / Benceno & 0.767 \\
Ciclohexano / 2,3,4-TMP & 0.983 & Tolueno / $m$, p-Xileno & 0.740 \\
Dimetilhexano / 2,3,4-TMP & 0.967 & Benceno / Tolueno & 0.712 \\
Dimetilhexano / o-Xileno & 0.939 & 2-Metil-Pentano / Ciclohexano & 0.624 \\
2,3,4-TMP / 2,2,4-TMP & 0.927 & Ciclohexano / o-Xileno & 0.501 \\
Dimetilhexano / 2,2,4-TMP & 0.926 & & \\
2,3,4-TMP / o-Xileno & 0.906 & & \\
Heptano / Tolueno & 0.899 & & \\
Heptano / o-Xileno & 0.892 & & \\
2-Metil-Pentano / 2,2,4-TMP & 0.824 & & \\
Benceno / m, p-Xileno & 0.843 & & \\
2-Metil-Pentano / 2,3,4-TMP & 0.840 & & \\
2-Metil-Pentano / Dimetilhexano & 0.832 & & \\
\hline
\end{tabular}

vehículo o condiciones del vehículo mismo. Por ejemplo, la corelación entre Benceno y Tolueno para vehículos que usaban gasolina Magna fue de 0.877 , mientras que para vehículos con gasolina Premium fue de 0.661. Esto se explora con más detalle en la siguiente sección.

Estos resultados permiten, por una parte, verificar la consistencia de los valores de concentración obtenidos al identificar correlaciones que es de esperarse que sean altas por la naturaleza química de las especies (p. ej., entre o-Xileno y $m, p$-Xileno) y correlaciones entre especies que la literatura reporta como características de emisiones vehiculares (p. ej., Benceno vs. Tolueno). Los valores de algunas de las correlaciones entre compuestos aromáticos como Benceno vs Tolueno y Etilbenceno vs o-Xileno (con $r^{2}$ de 0.712 y 0.783 , respectivamente) son similares a los valores reportados por estudios en donde se obtuvieron muestras de aire ambiente altamente influenciadas por emisiones vehiculares. Por ejemplo, $\mathrm{Na}$ et al. (2005) reportan para las mismas combinaciones valores de $r^{2}$ de 0.77 y 0.65 , respectivamente. Así mismo, Chan et al. (2002) reportan para las combinaciones Benceno vs Tolueno y Etilbenceno vs o-Xileno las siguientes $r^{2}$ : 0.729 y 0.992 , respectivamente; por otro lado, en este estudio se obtuvieron para esas mismas combinaciones valores de $r^{2}$ de 0.712 y 0.783 , respectivamente.
Así, los resultados presentados indican, en lo general, una consistencia con lo reportado en la literatura.

\section{Análisis estadístico}

Se realizaron una serie de análisis de medias y análisis de varianza (ANOVA en un solo sentido acompañado de pruebas de Tukey-Kramer) para determinar la relación entre los valores de concentración obtenidos para cuatro categorías predefinidas: año-modelo, kilometraje, marca y tipo de gasolina y poder hacer inferencias respecto al comportamiento de las concentraciones reportadas. Si bien no se probó que los datos experimentales cumplían con las suposiciones en las que se basa el ANOVA, se trabajó bajo el argumento de que la prueba $F$ es robusta. Cabe resaltar que para estos análisis estadísticos solamente se utilizaron los resultados obtenidos para aquellos vehículos monitoreados en ambas campañas.

En la tabla 5 se presentan las concentraciones promedio cuando se clasificaron los resultados de acuerdo al tipo de gasolina empleada por los vehículos muestreados. Se puede observar que, excepto para el Benceno y el Etilbenceno, el valor de concentración es mayor para los vehículos con gasolina Premium. Sin embargo, solamente los niveles de 2,2,4-Trimetilpentano, Dimetilhexano y 
DOI: http://dx.doi.org/10.22201/fi.25940732e.2010.11n3.029

Mendoza-Domínguez A., León-Romero M.A. y Caballero-Mata P.

2,3,4-Trimetilpentano son estadísticamente diferentes entre las dos clasificaciones. En el caso del 2,2,4-Trimetilpentano, la concentración obtenida para vehículos con gasolina Premium es aproximadamente 7.7 veces mayor que la encontrada para los vehículos que usaron gasolina Magna. El Dimetilhexano y el 2,3,4-Trimetilpentano no fueron detectados en las muestras de vehículos que empleaban gasolina Magna.

En la tabla 6 se presentan las concentraciones promedio cuando los datos se clasificaron por el kilometraje de los automóviles muestreados. Se observa que, salvo por el 2,2,4-Trimetilpentano, las concentraciones promedio de los compuestos analizados tienen su valor más alto en los vehículos con kilometraje entre 0 y $15,000 \mathrm{~km}$. Asimismo, las concentraciones de todos los compuestos, excepto Benceno y 2,2,4-Trimetilpentano, fueron estadísticamente iguales. Cabe notar que no se detectó ni Dimetilhexano ni 2,3,4-Trimetilpentano en las muestras de los vehículos con kilometraje entre 45,000 y $60,000 \mathrm{~km}$. En el caso particular del 2,2,4-Trimetilpentano, la concentración reportada para el intervalo intermedio de kilometraje $(15,000-30,000 \mathrm{~km})$ es aproximadamente 6.6 veces más alta que los vehículos con mayor kilometraje. En cambio, las concentraciones entre las otras dos primeras categorías es muy parecida (141.7 ng/ml vs $185.0 \mathrm{ng} / \mathrm{mL}$ ). Para el Benceno los valores de concentración para las tres categorías son cercanos, resultando la concentración reportada para vehículos con bajo kilometraje apenas 1.7 veces más grande que los vehículos con intervalo 15,000-30,000 $\mathrm{km}$ y 1.5 veces la de los vehículos con kilometraje de 45,000-60,000 km.

Considerando que en la muestra de vehículos empleada en este estudio los que utilizan Magna son también aquellos con el mayor kilometraje y, de acuerdo a los resultados presentados en la tabla 5 estos vehículos presentan baja o nula concentración de 2,2,4-Trimetilpentano, Dimetilhexano y 2,3,4-Trimetilpentano, se puede inferir que los niveles de contaminantes observados son una función débil del kilometraje recorrido por los vehículos. Es decir, los vehículos muestreados en los dos primeros intervalos de kilometraje son

Tabla 5. Concentraciones promedio $(\mathrm{ng} / \mathrm{mL})$ por tipo de gasolina

\begin{tabular}{ccc}
\hline & magna $(\mathrm{N}=8)$ & Premium $(\mathrm{N}=12)$ \\
\hline Benceno & $33.8 \mathrm{a}^{*}$ & $28.8 \mathrm{a}$ \\
Tolueno & $51.7 \mathrm{a}$ & $62.2 \mathrm{a}$ \\
Etilbenceno & $10.8 \mathrm{a}$ & $5.6 \mathrm{a}$ \\
o-Xileno & $17.8 \mathrm{a}$ & $18.5 \mathrm{a}$ \\
2,2,4-Trimetilpentano & $24.1 \mathrm{a}$ & $185.0 \mathrm{~b}$ \\
Dimetilhexano & $\mathrm{MLD} \mathrm{a}$ & $122.9 \mathrm{~b}$ \\
2,3,4-Trimetilpentano & $\mathrm{MLD} \mathrm{a}$ & $102.5 \mathrm{~b}$ \\
\hline
\end{tabular}

* En una misma fila, valores con letras diferentes son significativamente diferentes (Prueba de Tukey, p < 0.05). N: Número de datos empleados en la clasificación. MLD: Menor al límite de detección.

Tabla 6. Concentraciones promedio $(\mathrm{ng} / \mathrm{mL})$ por intervalos de kilometrajes

\begin{tabular}{cccc}
\hline & $0-15,000 \mathrm{~km}$ & $15,000-30,000 \mathrm{~km}$ & $45,000-60,000 \mathrm{~km}$ \\
$(\mathrm{~N}=8)$ & $(\mathrm{N}=6)$ & $(\mathrm{N}=6)$ \\
\hline Benceno & $39.9 \mathrm{a}$ & $23.4 \mathrm{~b}$ & $26.2 \mathrm{ab}$ \\
Tolueno & $86.5 \mathrm{a}$ & $37.7 \mathrm{a}$ & $40.4 \mathrm{a}$ \\
Etilbenceno & $8.4 \mathrm{a}$ & $5.9 \mathrm{a}$ & $8.8 \mathrm{a}$ \\
o-Xileno & $24.0 \mathrm{a}$ & $16.5 \mathrm{a}$ & $12.3 \mathrm{a}$ \\
2,3,4-Trimetilpentano & $141.7 \mathrm{ab}$ & $185.0 \mathrm{a}$ & $28.1 \mathrm{~b}$ \\
Dimetilhexano & $105.7 \mathrm{a}$ & $104.8 \mathrm{a}$ & $\mathrm{MLD} \mathrm{b}$ \\
2,3,4-Trimetilpentano & $91.1 \mathrm{a}$ & $83.5 \mathrm{a}$ & $\mathrm{MLD} \mathrm{b}$ \\
\hline
\end{tabular}

* Ver Tabla 5 para detalles de la nomenclatura usada. 
preferentemente autos que usan gasolina Premium y sus perfiles son (estadísticamente) prácticamente los mismos (con excepción del Benceno), mientras que los del tercer intervalo son preferentemente vehículos que usan gasolina Magna y las diferencias observadas respecto a los otros dos intervalos reflejan más bien las diferencias en uso de gasolina y no necesariamente el kilometraje del vehículo al estar asociado éste con el 2,2,4-Trimetilpentano, el 2,3,4- Trimetilpentano y el Dimetilhexano.

La tabla 7 presenta las concentraciones promedio cuando se realizó la clasificación de los automóviles por marca. Se observa que, en general, las concentraciones más altas se presentan en los vehículos de la marca Nissan. Para los cuatro primeros compuestos, las concentraciones resultaron estadísticamente iguales entre las diferentes marcas. Para el caso del 2,2,4-Trimetilpentano, las concentraciones reportadas para los vehículos marca Peugeot, Renault y Chevrolet son estadísticamente iguales, mientras que las concentraciones entre los vehículos Nissan, Peugeot y VW son estadísticamente iguales. Una situación parecida se presenta para las concentraciones reportadas para Dimetilhexano y 2,3,4-Trimetilpentano, con la diferencia que las emisiones de los vehículos VW también es estadísticamente similar a la de los vehículos Chevrolet y Peugeot.

Al igual que en los casos anteriores, en estos resultados se puede ver la influencia del tipo de gasolina. Esto debido a que los vehículos monitoreados de marcas Nissan, Peugeot y VW en su mayoría utilizaban gasolina Premium, no así los vehículos marca Chevrolet, que en su totalidad usaban gasolina Magna. En otras palabras, los niveles de emisión también son una función débil de la marca del vehículo, siendo el tipo de gasolina el que aparentemente influye más en los resultados.

Finalmente, en la tabla 8 se aprecian las concentraciones promedio de las especies clasificadas por el añomodelo de los automóviles usados durante las

Tabla 7. Concentraciones promedio $(\mathrm{ng} / \mathrm{mL})$ por marca de vehículo

\begin{tabular}{|c|c|c|c|c|c|}
\hline & $\begin{array}{c}\text { Chevrolet } \\
(\mathrm{N}=10)\end{array}$ & $\begin{array}{l}\text { Nissan } \\
(\mathrm{N}=4)\end{array}$ & $\begin{array}{c}\text { Peugeot } \\
(\mathrm{N}=2)\end{array}$ & $\begin{array}{c}\text { Renault } \\
(\mathrm{N}=2)\end{array}$ & $\begin{array}{c}\mathrm{VW} \\
(\mathrm{N}=2)\end{array}$ \\
\hline Benceno & $32.7 \mathrm{a}$ & $35.1 \mathrm{a}$ & $28.5 \mathrm{a}$ & $19.5 \mathrm{a}$ & $26.5 a$ \\
\hline Tolueno & $49.9 \mathrm{a}$ & $109.2 \mathrm{a}$ & $40.7 \mathrm{a}$ & $32.5 \mathrm{a}$ & $39.2 \mathrm{a}$ \\
\hline Etilbenceno & $8.71 \mathrm{a}$ & $10.2 \mathrm{a}$ & $5.2 \mathrm{a}$ & MLD & $8.15 \mathrm{a}$ \\
\hline o-Xileno & $18.3 \mathrm{a}$ & $27.2 \mathrm{a}$ & $11.5 \mathrm{a}$ & $9.4 \mathrm{a}$ & $15.6 \mathrm{a}$ \\
\hline 2,2,4-Trimetilpentano & $48.6 \mathrm{~b}$ & $251.0 \mathrm{a}$ & $174.5 \mathrm{ab}$ & $35.5 \mathrm{~b}$ & $251.5 \mathrm{a}$ \\
\hline Dimetilhexano & $20.0 \mathrm{~b}$ & $172.2 \mathrm{a}$ & $117.0 \mathrm{ab}$ & MLD & $176.0 \mathrm{ab}$ \\
\hline 2,3,4- Trimetilpentano & $16.6 \mathrm{~b}$ & $141.5 \mathrm{a}$ & $102.0 \mathrm{ab}$ & MLD & $147.0 \mathrm{ab}$ \\
\hline
\end{tabular}

* Ver tabla 5 para detalles de la nomenclatura usada.

Tabla 8. Concentraciones promedio $(\mathrm{ng} / \mathrm{mL})$ por año modelo

\begin{tabular}{ccccc}
\hline & 1999 & 2000 & 2004 & 2005 \\
$(\mathrm{~N}=2)$ & $(\mathrm{N}=4)$ & $(\mathrm{N}=8)$ & $24.6 \mathrm{~b}=6)$ & $43.8 \mathrm{a}$ \\
\hline Benceno & $24.0 \mathrm{ab}$ & $27.2 \mathrm{ab}$ & $38.9 \mathrm{~b}$ & $101.1 \mathrm{a}$ \\
Tolueno & $33.8 \mathrm{ab}$ & $43.7 \mathrm{ab}$ & $4.41 \mathrm{a}$ & $11.1 \mathrm{a}$ \\
Etilbenceno & $7.55 \mathrm{a}$ & $9.40 \mathrm{a}$ & $17.4 \mathrm{a}$ & $25.3 \mathrm{a}$ \\
o-Xileno & $\mathrm{MLD} \mathrm{b}$ & $18.5 \mathrm{a}$ & $175.3 \mathrm{a}$ & $140.1 \mathrm{a}$ \\
2,2,4-Trimetilpentano & $36.0 \mathrm{a}$ & $24.2 \mathrm{a}$ & $103.6 \mathrm{a}$ & $107.7 \mathrm{a}$ \\
Dimetilhexano & MLD b & MLD b & $83.3 \mathrm{a}$ & $93.8 \mathrm{a}$ \\
$2,3,4-$ Trimetilpentano & MLD b & & &
\end{tabular}

* Ver tabla 5 para detalles de la nomenclatura usada. 
campañas de monitoreo. Es notable que las concentraciones de los vehículos modelo 2005 presentan los valores más altos entre las categorías, excepto para el caso del 2,2,4- Trimetilpentano.

En este análisis, las concentraciones de Benceno y Tolueno resultaron ser estadísticamente diferentes entre diversos años modelo. Sin embargo, una vez más se observan las principales diferencias entre las concentraciones de 2,2,4-Trimetilpentano, 2,3,4-Trimetilpentano y Dimetilhexano. Las concentraciones son más altas en los modelos 2004 y 2005, respecto a los vehículos modelos 1999 y 2000. En este estudio, los vehículos modelo 2004-2005 usaban en su mayoría gasolina Premium. Con esto se puede nuevamente inferir que la alta concentración de los tres compuestos citados anteriormente es debida primordialmente a la gasolina empleada $y$ no tanto al año-modelo del vehículo.

Un complemento a la información arriba presentada serían las emisiones de los contaminantes expresados en términos de gramos emitidos durante el periodo que duró la prueba. Para estimar dichas emisiones se puede usar información de flujo medido en el escape, o bien, estimar el flujo con lecturas de la velocidad del motor (revoluciones por minuto), cilindrada y temperatura de los gases de escape. En cualquier caso, esta información no se registró para las pruebas efectuadas, por lo que no se puede hacer un buen estimado de las mismas. Sin embargo, la información aquí presentada sigue siendo útil al proporcionar una base cuantificable de concentraciones de contaminantes atmosféricos en el flujo de escape que posteriormente pueden ser usadas para estimar emisiones de vehículos una vez conocidas las condiciones particulares de funcionamiento.

\section{Discusión}

\section{Relación entre compuestos}

$\mathrm{Al}$ igual que las correlaciones, las proporciones entre diversos COV pueden ser muy útiles para identificar las fuentes emisoras de este tipo de especies (Gee et al., 1998). Dentro del amplio intervalo de razones entre concentraciones promedio de COVs que se pueden emplear, se destaca el uso frecuente de las relaciones de los aromáticos; en especial del grupo conformado por Benceno, Tolueno, Etilbenceno y Xileno, comúnmente denominado como BTEX.

La tabla 9 presenta un resumen de razones calculadas para BTEX, basado en datos obtenidos de varios estudios enfocados al análisis de emisiones de parque vehicular y cómo se comparan dichos valores con los obtenidos en este estudio.

Se puede observar que los valores obtenidos en este trabajo caen en el intervalo de valores reportados por otros, con la relación X:B un poco inferior al valor mínimo reportado en las referencias bibliográficas consultadas. Los valores obtenidos en el estudio de Singer et al. (1999) se refieren por separado, ya que dicho trabajo estuvo particularmente enfocado a la caracterización de emisiones por arranques en frío. Las diferencias encontradas pueden deberse a muchos factores, aunque principalmente se puede deber al tipo de combustible empleado.

No obstante, los resultados son valiosos al tenerse indicadores que se pueden emplear en otros estudios que analicen flotas vehiculares mexicanas como proporciones que pueden trazar su origen a emisiones por vehículos en arranque en frío.

Tabla 9. Intervalos de las razones de BTEX obtenidos de la literatura

\begin{tabular}{ccccc}
\hline & Valores reportados en la literatura $\left(^{*}\right)$ & & Singer et al. \\
Razón & Mínimo & Máximo & (1999) & $\begin{array}{c}\text { Promedio } \\
\text { (este trabajo) }\end{array}$ \\
\hline T:B & 1.75 & 4.0 & 4.0 & 1.9 \\
E:B & 0.3 & 0.6 & 0.6 & 0.6 \\
X:B & 1.6 & 3.4 & 3.4 & 1.5 \\
\hline
\end{tabular}

(*) De acuerdo a valores reportados por: Fujita et al. (1992), Sweet et al. (1992), Scheff et al. (1993), Kenski et al. (1995), Zielinska et al. (1996), Gee et al. (1998), Mujica et al. (1998), Singer et al. (1999), Chan et al. (2002), Doskey et al. (2002), Na et al. (2004, 2005). 
DOI: http://dx.doi.org/10.22201/fi.25940732e.2010.11n3.029

Emisiones de compuestos orgánicos volátiles durante arranques en frío de automóviles ligeros

\section{Reactividad}

El término de reactividad es utilizado frecuentemente para denotar el potencial de formación de ozono de un COV. El análisis de la reactividad de la emisión de una fuente particular es importante, ya que el impacto de la emisión no está vinculada exclusivamente a la masa emitida, sino a la combinación entre reactividad y masa emitida (Russell et al., 1995). La reactividad total de una emisión pudiera no necesariamente estar gobernada por la especie más abundante. Esta información es útil cuando se va a determinar el impacto global de la emisión en la formación de ozono y en la definición de estrategias de control.

Se han propuesto varias escalas de reactividad de los COVs. En este estudio se empleó la escala propuesta por Chameides et al. (1992) conocida como "concentración propileno equivalente" o "propy-equiv". Esta es una escala de normalización simple, la cual se basa en transformar la concentración de un determinado COV a su equivalente reactivo de propileno al relacionar la velocidad de reacción de dicho compuesto con el radical libre $\mathrm{HO}$ ' respecto a la velocidad de reacción del compuesto de referencia (en este caso, el propileno):

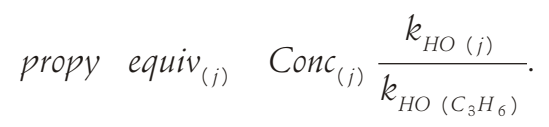

En la ecuación (1) $\operatorname{Conc}_{(j)}$ es la concentración de la especie $j$ (en ppbC), $k_{H O(i)}$ es la constante de velocidad para la reacción entre la especie j y el radical $\mathrm{HO} \cdot k_{\mathrm{HO}(\mathrm{C} 3 \mathrm{H} 6)}$ es la constante de velocidad para la reacción entre el propileno y el radical $\mathrm{HO}^{\bullet}$. En otras palabras, la concentración propileno equivalente es la concentración de propileno (en ppbC) requerida para alcanzar una velocidad de oxidación de carbono igual al del COV $j$.

Los valores promedio de emisión obtenidos, posterior a su cambio de unidades de $\mu \mathrm{g} / \mathrm{m}^{3}$ a $\mathrm{ppbC}$, fueron evaluados de acuerdo a la ecuación 1 y reordenados de acuerdo a su concentración propilieno equivalente (tabla 10). Las constantes de velocidad de reacción fueron obtenidas de Carter (2008); se emplearon las constantes de reacción evaluadas a $300 \mathrm{~K}$. El Dimetilhexano, compuesto que se detectó con la mayor concentración promedio, fue también el compuesto con la mayor reactividad relativa. Es notorio los cambios que reportaron el 2,2,4-Trimetilpentano que pasó de ser la segunda especie en concentración a la quinta en reactividad y el 1,2,4-Trimetilbenceno de ser la doceava especie en concentración a ser la tercera en reactividad. De igual forma el $m, p$-Xileno pasó de ser la especie con menor concentración a la séptima en reactividad. Como referencia, en un estudio realizado en la ciudad de Atlanta, EE.UU. (NRC, 1991) se reportó la reactividad propileno equivalente en muestras de aire

Tabla 10. Concentraciones promedio y concentraciones propileno equivalente obtenidas para las especies identificadas en los muestreos de las dos campañas

\begin{tabular}{cccc}
\hline \multicolumn{2}{c}{ Especies ordenadas por concentración } & \multicolumn{2}{c}{ Especies ordenadas por reactividad HO } \\
\hline Compuesto & Concentración $(\mathrm{ppmC})$ & Compuesto & Propy-Equiv (ppmC) \\
\hline Dimetilhexano & 314.9 & Dimetilhexano & 103.8 \\
2,2,4-Trimetilpentano & 305.5 & $2,3,4$-Trimetilpentano & 68.0 \\
2,3,4-Trimetilpentano & 267.9 & $1,2,4$-Trimetilbenceno & 53.3 \\
Ciclohexano & 173.6 & Ciclohexano & 46.7 \\
Tolueno & 113.4 & $2,2,4$-Trimetilpentano & 39.7 \\
n-Pentano & 102.9 & o-Xileno & 29.8 \\
2-Metilpentano & 101.0 & m,p-Xileno & 24.5 \\
$n$-Hexano & 78.7 & Tolueno & 24.3 \\
Benceno & 60.8 & 2 -Metilpentano & 20.2 \\
o-Xileno & $n$-Hexano & 15.9 \\
$n$-Heptano & 57.0 & $n$-Pentano & 15.1 \\
1,2,4-Trimetilbenceno & 46.1 & $n$-Heptano & 12.1 \\
Etilbenceno & 42.7 & Etilbenceno & 9.8 \\
m,p-Xileno & 36.2 & Benceno & 2.9 \\
\hline
\end{tabular}


ambiente. En dicho estudio, compuestos como el $m, p$-Xileno, Tolueno y 1,2,4-Trimetilbenceno se encontraron entre los diez primeros de la mencionada lista (lugares 3, 6 y 8, respectivamente). Finalmente, dado que los resultados del análisis estadístico revelaron que las emisiones de los vehículos que emplean gasolina Premium reportan los mayores niveles de Dimetilhexano y 2,2,4-Trimetilpentano, esto implicaría que la reactividad de dichas emisiones es mayor que las obtenidas de los vehículos que emplean gasolina Magna.

\section{Conclusiones}

El análisis químico realizado a las muestras de gases de escape de los vehículos usados en este estudio, indicó que las especies con mayor concentración fueron: Dimetilhexano, 2,2,4 Trimetilpentano y 2,3,4 Trimetilpentano. Al hacer un análisis estadístico de los datos se encontró que la presencia o alta concentración de dichas especies químicas es producto principalmente de la diferencia de gasolinas empleadas, estando los compuestos antes mencionados relacionados con la gasolina Premium.

Así, el kilometraje recorrido, el año-modelo o la marca del vehículo no afecta en gran media al perfil químico observado de las emisiones; solamente el tipo de gasolina es determinante. Al realizar una estimación del potencial de formación de ozono para los compuestos detectados, se encontró que las mezclas más reactivas son las que incluyen precisamente Dimetilhexano y 2,3,4-Trimetilpentano, indicando que los vehículos que emplean gasolina Premium emiten mezclas más reactivas. Finalmente, se obtuvo que en promedio la proporción de T:B, E:B y X:B en los gases emitidos es de 1.9, 0.6 y 1.5, con una buena correlación $\left(r^{2}>0.5\right)$ para la primer y tercer proporción. Estos últimos resultados proporcionan información valiosa para estudios que trazan emisiones a partir de concentraciones de contaminantes en aire ambiente, al permitir tener indicadores para identificar emisiones de arranque en frío que pudieran ser característicos de una porción de la flota vehicular mexicana.

\section{Agradecimientos}

Agradecemos al Laboratorio de Calidad del Aire del Centro de Calidad Ambiental del Tecnológico de Monterrey, Campus Monterrey, el apoyo brindado para realizar estes estudio. Asimismo, agradecemos a la Dirección de Investigación y Postgrado del Campus Monterrey su apoyo canalizado a través de la Cátedra de
Investigación en Ingeniería de la Contaminación Atmosférica (CAT-186).

\section{Referencias}

Brocco D., Fratancangeli R., Lepore L., Preticca M. y Ventrone I. Determation of Aromatic Hydrocarbons in Urban Air of Rome. Atmos. Environ., (31):557-566. 1997.

Carter W.P.L. Development of the SAPRC-07 Chemical Mechanism and Updated Ozone Reactivity Scales. Final report to the California Air Resources Board (Contract No. 03-318), prepared by the University of California, Riverside, pp. 369. 2008.

Chameides W.L., Fehsenfeld F., Rodgers M.O., Cardelino C., Martinez J., Parrish D., Lonneman W., Lawson D.R., Rasmussen R.A., Zimmerman P., Greenburg J., Middleton P. y Wang T. Ozone Precursor Relationships in the Ambient Atmosphere. J. Geophys. Res., (97):6037-6055. 1992.

Chan C.Y., Chan L.Y., Wang X.M., Liu Y.M., Lee S.C., Zou S.C., Sheng G.Y. y Fu J.M. Volatile Organic Compounds in Roadside Microenvironments of Metropolitan Hong Kong. Atmos. Environ., (36):2039-2047. 2002.

Cheng I., Fu I., Angle R. y Sandhu H. Seasonal Variation of Volatile Organic Compounds in Edmonton, Alberta. Atmos. Environ., (31):239-246. 1997.

Corvalan R.M., Galecio J.I. y Sallm J.A. Effectiveness in the Use of Natural Gas for the Reduction of Atmospheric Emissions: Case Study-Industrial Sector in the Metropolitan Region of Santiago, Chile. J. Air \& Waste Manage. Assoc., (53):971-975. 2000.

Derwent R., Davies T., Delaney T., Dollard G., Field R., Dumitrean P., Nason P., Jones B. y Pepler S. Analysis and Interpretation of the Continuous Hourly Monitoring Data for 26 C2-C8 Hydrocrabons at 12 United Kingdom sites during 1996. Atmos. Environ., (34):297-312. 2000.

Devore J.L. Probabilidad y Estadística para Ingeniería y Ciencias.

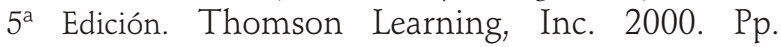
522-523.

Díaz L., Schifter I., Rodríguez R., Avalos S., López G. y López-Salinas E. Long Term Efficiency of Catalytic Converters Operating in México City. J. Air \& Waste Manage. Assoc., (51):725-732. 2001.

Doskey P.V., Fukui Y., Sultan M., Maghraby A.A. y Taher A. Source Profiles for Nonmethane Organic Compounds in the Atmosphere of Cayro, Egypt. J. Air \& Waste Manage. Assoc., (49):814-822. 2002.

ERG, Acosta y Asociados y TransEngineering Mexico National Emissions Inventory, 1999: Six Northern States. Final Report No. 3393-00-011-002, Sacramento, CA. 2004.

Gamas E.D., Diaz L., Rodríguez R., López-Salinas E., Schifter I. y Ontiveros L. Exhaust Emissions from Gasoline- 
DOI: http://dx.doi.org/10.22201/fi.25940732e.2010.11n3.029

Emisiones de compuestos orgánicos volátiles durante arranques en frío de automóviles ligeros

and LPG-Powered Vehicles Operating at the Altitude of México City. J. Air \& Waste Manage. Assoc., (49):1179-1189. 1999.

Gee I.L. y Sollars C.J. Ambient Air Levels of Volatile Organic Compounds in Latin American and Asian Cities. Chemosphere, (36):2497-2506. 1998.

Graham L.A., Noseworthy L., Fugler D., O'Leary K., Karman D. y Grande C. Contribution of Vehicle Emissions from an Attached Garage to Residential Indoor Air Pollution Levels. J. Air \& Waste Manage. Assoc., (54):563-568. 2004.

Grosjean E., Grosjean D. y Ramussen R. Ambient Concentrations, Sources, Emission Rates and Photochemical Reactivity of C2-C10 Hydrocarbons in Porto Alegre, Brazil. Environ. Sci. Technol., (32):2061-2069. 1998.

Heywood J.B. Internal Combustion Engine Fundamentals. Mc Graw-Hill, New York. 1988. Pp. 930.

Ho J. y Winer A.M. Effects of Fuel Type, Driving Cycle, and Emission Status on In Use Vehicle Exhaust Reactivity. J. Air \& Waste Manage. Assoc., (48):592-603. 1998.

Kenski D.M., Wadden R.A., Scheff P.A. y Lonneman W.A. Receptor Modeling Approach to VOC Emission Inventory Validation. J. Environ. Eng., (121):483-491. 1995.

Mendoza A., Gutiérrez A.A. y Pardo E.I. Volatile Organic Compounds in the Downtown Area of Mexicali, Mexico During the Spring of 2005: Analysis of Ambient Data and Source-Receptor Modeling. Atmósfera, (22):195-217. 2008.

Mugica V., Vega E., Arriaga J.L. y Ruiz M.E. Determination of Motor Vehicle Profiles for Non-Methane Organic Compounds in the México City Metropolitan Area. J. Air \& Waste Manage. Assoc., (48):1060-1068. 1998.

Mugica V., Vega E., Sanchez G., Reyes E., Arriaga J. L., Chow J., Watson J. y Egami R. Volatile Organic Compounds Emissions from Gasoline and Diesel Powered Vehicle. Atmósfera, (14):29-37. 2001.

Mugica V., Ruiz M.E., Watson J. y Chow J. Volatile Organic Compounds in México City Atmosphere: Levels and Sources Apportionment. Atmósfera, (16):15-27. 2003.

Mukerjee S., Smith L.A., Norris G.A., Morandi M.T., Gonzales M., Noble C.A., Neas L.M. y Özkaynak A.H. Field Method Comparison Between Passive Air Samplers and Continuous Monitors for VOC's and $\mathrm{NO}_{2}$ in El Paso, Texas. J. Air \& Waste Manage. Assoc., (54):307-319. 2004.

$\mathrm{Na}$ K. y Kim Y. Seasonal Characteristics of Ambient Volatile Organic Compounds in Seoul, Korea. Atmos. Environ., (35):2603-2614. 2001.

Na K., Kim Y.P., Moon I. y Moon K.C. Chemical Composition of Major VOC Emission Sources in the Seoul Atmosphere. Chemosphere, (55):585-594. 2004.

Na K., Mon K.C. y Kim Y.P. (2005). Source Contribution to Aromatic VOC Concentration and Ozone Formation
Potential in the Atmosphere of Seoul. Atmos. Environ., (39):5517-5524.

NRC (National Research Council) Rethinking the Ozone Problem in Urban and Regional air Pollution. National Academy Press, Washington, D.C. pp. 500. 1991.

Quesada T. Comparación del Desempeño Ambiental del Sector Transporte en Nuevo León, a través de Indicadores Ambientales y Energéticos. Tesis (maestría en ciencias).

México. Instituto Tecnológico y de Estudios Superiores de Monterrey, Campus Monterrey. 2003.

Russell A.G., Milford J.B., Bergin M.S., McBride S., McNair L., Yang Y., Stockwell W. y Croes B.E. Urban Ozone Control and Atmospheric Reactivity of Organic Gases. Science, (269):491-495. 1995.

Seinfeld J.H. y Pandis S.N. Atmospheric Chemistry and Physics of Air Pollution: From Air Pollution to Climate Change. Wiley-Interscience. New York. 1998. Pp.1326.

Scheff P.A. y Wadden R.A. Receptor Modeling of Volatile Organic Compounds. 1. Emission Inventory and Validation. Environ. Sci. Technol., (27):617-625. 1993.

Singer B.C., Kirchstetter T.W., Harley R.A., Kendall G.R. y Hesson J.M. A Fuel-Based Approach to Estimating Motor Vehicle Cold-Start Emissions. J. Air \& Waste Manage. Assoc., (49):125-135. 1999.

Sweet C.W. y Vertmette S.J. Toxic Volatile Organic Compounds in Urban Air in Illinois. Environ. Sci. Technol., (26):165-173. 1992.

Thijsse T., van Oss R.F. y Lenschow. P. Determination of source Contributions to Ambient Organic Compound Concentrations in Berlin. J. Air \& Waste Manage. Assoc., (49):1394-1404. 1999.

US EPA (United States Environmental Protection Agency). Federal Test Procedure Review Project: Preliminary Technical Report, EPA 420/R-93-007. Office of Air \& Radiation, pp. 203. 1993.

US EPA (United States Environmental Protection Agency). National Air Quality and Emissions Trends Report, EPA 454/R-01-004. Research Triangle Park, NC, pp. 238. 2001.

Varshney C.K. y Padhy P.K. Total Volatile Organic Compounds in the Urban Environment of Delhi. J. Air \& Waste Manage. Assoc., (48):448-453. 1998.

Vega E., Mugica V., Carmona R. y Valencia E. Hydrocarbon Source Apportionment in Mexico City Using the Chemical Mass Balance Receptor Model. Atmos. Environ., (34): 4121-4129. 2000

Vukovich F. Weekday/Weekend Differences in OH Reactivity with VOCs and CO in Baltimore, Maryland. J. Air \& Waste Manage. Assoc., (50):1843-1851. 2000.

Watson J.G., Chow J.C. y Fujita E.M. Review of Volatile Organic Compound Source Apportionment by Chemical Mass Balance. Atmos. Environ., (35):1567-1584. 2001. 
DOI: http://dx.doi.org/10.22201/fi.25940732e.2010.11n3.029

Mendoza-Domínguez A., León-Romero M.A. y Caballero-Mata P.

Zielinska B., Sagebiel J.C., Harshfield G., Gertler A.W. y Pierson W.R. Volatile Organic Compounds up to $\mathrm{C}_{20}$ Emitted from Motor Vehicles; Measurement Methods. Atmos. Environ., (30):2269-2286. 1996.
Zielinska B., Sagebiel J, McDonald J.D., Whitney K. y Lawson D.R. Emission Rates and Comparative Chemical Composition from Selected In-Use and Gasoline-Fueled Vehicles. J. Air \& Waste Manage. Assoc., (54):1138-1150. 2004.

Semblanza de los autores

Alberto Mendoza-Dominguez. Es profesor-investigador del Departamento de Ingeniería Química del Tecnológico de Monterrey, Campus Monterrey. Obtuvo su grado de doctor en ingeniería ambiental del Instituto Tecnológico de Georgia, EE.UU., en 2001. Es miembro del Sistema Nacional de Investigadores (SNI) desde el 2002. Sus áreas de investigación están relacionadas principalmente con la ingeniería de la contaminación atmosférica.

Marco Antonio León-Romero. Obtuvo la licenciatura en Ingeniería Química en la Benemérita Universidad Autónoma de Puebla, y el grado de maestro en ciencias con especialidad en Sistemas Ambientales del Tecnológico de Monterrey (Campus Monterrey). Actualmente labora en el Centro del Agua para América Latina y el Caribe (CAALCA).

Porfirio Caballero-Mata. Es profesor-investigador del Centro de Calidad Ambiental del Tecnológico de Monterrey, Campus Monterrey. Obtuvo su grado de doctor en química de la Universidad Estatal de Louisiana, EE.UU., en 1981. Sus áreas de investigación están relacionadas principalmente con la química ambiental. 